Materials Science Forum Vols. *** (2004) pp.722-726

online at http://scientific.net

C 2004 Trans Tech Publications, Switzerland

\title{
Manufacturability of Fractal Geometry
}

\author{
Y.C. Yeung ${ }^{1, a}$ and K.M. Yu ${ }^{1, b}$ \\ ${ }^{1}$ Department of Industrial \& Systems Engineering, The Hong Kong Polytechnic University, Hung Hom, \\ Kowloon, Hong Kong \\ amfposy@polyu.edu.hk, ${ }^{\mathrm{b}} \mathrm{mfkmyu@inet.polyu.edu.hk}$
}

Keywords: Fractal geometry, Layer manufacturing, Manufacturability

\begin{abstract}
Nowadays more and more aesthetic product developments, assemblage and decoration designs are taking aesthetically appealing forms of natural objects such as rough terrain, ripples on lakes, coastline and seafloor topography. They are mathematical definable via fractal geometry theory and emerge to attract a lot of attention. However, not many methods for manufacturing of fractal objects have been reported in the literature and no previous research papers concern the manufacturability of fractal geometry. The paper will, thus, give a tentative classification and nomenclature of fractal geometry. Then, a state-of-the-art overview of manufacturing techniques is presented. By bridging the gap between fractal geometry and manufacturing, those processes that are promising to manufacture the three dimensional (3D) fractal objects will be highlighted. Afterward, a brief overview of limitation of those processes will be discussed.
\end{abstract}

\section{Introduction}

Analyzing the manufacturability of the proposed design has become an important step in the design stage. Recently, visibility is widely used to many classes of problem in design and manufacturing. Visibility [1] is viewpoint-to-region and it is meaningful when a pair consisting of a source and a target is considered. Geometrically, the target (or the workpiece) can be a point, a line segment, a curve segment, a planar polygon, or a surface patch. Similarly, the source (or the effector) can be modeled hierarchically by its topological dimensionalities: 0D points, 1D straight lines and curves, and 2D planes and surfaces.

This paper will base on visibility to summarize an interdisciplinary approach to classify those processes that can manufacture fractal geometry. The manufacturability of a fractal geometry defines fractal objects that can be manufacturable by certain process. Emphasis is placed on the two fundamental questions that arise concerning every type of manufacturing process: (a) Can fractal be manufactured by existing conventional process? (b) Is fractal only manufacturable by LM technology? The survey in this paper attempts to answer the above questions by considering the manufacturability of fractal objects, as well as to explore the limits of the technologies. It will go on to discuss how the promising techniques can better serve the requirements of fractal geometry fabrication.

\section{Brief Review of Fractal Geometry}

Fractal is according to the definition given by B. Mandelbrot [2], a set with a Haussdorf-Bessicovitch dimension strictly higher than its topological dimension. This definition covers several geometries that are self-similar under several scales.

Two main types of fractals will be considered including iterative function system (IFS) fractals (e.g., self-intersecting fractal objects, simply connected fractal objects, multiply connected fractal objects, natural objects, area filling fractal objects, etc.) and complex fractals (e.g., quaternion Mandelbrot set, 
quaternion Julia set, filled-in Julia set, contour Julia set, dust Julia set, Dendrite Julia set, etc.) Barnsley and Demko [3] first propose the iterative algorithm method called IFS.

Minkowski, Hilbert, Peano curves, Sierpinski gasket, the Sierpinski carpet and the Koch Island are typical examples that could be found in the literature of fractals. All these intricate geometries are built using an IFS method which can be expressed mathematically with Eq.1, Eq. 2 and Eq. 3.

$$
A_{i}=W\left[A_{i-1}\right] \quad i=0,1, \ldots, \infty
$$

where $W$ is the Hutchinson's geometrical transformation algorithm

$W[A]=w_{1}(A) \cup w_{2}(A) \cup \ldots \cup w_{n}(A)$

and $w$ being an affine transformation. A recursive affine transformation is

$$
\left(\begin{array}{l}
x_{n+1} \\
y_{n+1}
\end{array}\right)=\left(\begin{array}{ll}
a & b \\
c & d
\end{array}\right)\left(\begin{array}{l}
x_{n} \\
y_{n}
\end{array}\right)+\left(\begin{array}{l}
e \\
f
\end{array}\right)
$$

After an infinite number of iterations, the initial set $A_{0}$, or initiator, which started the iterative algorithm, is transformed into the set $A$ that is the fractal. Each intermediate state $A_{i}$ is called prefractal. The complex fractals are generated and represented by complex set mappings and picture elements (pixels) respectively. For example, B. Mandelbrot (1924-,) a brilliant Polish mathematician, had developed and popularized a relatively novel class of mathematical functions know as Mandelbrot set. The function is defined by a simple recurrence formulation in the complex plane [2], mathematically,

$$
\mathrm{z}_{\mathrm{n}}=\mathrm{z}_{n-1}^{2}+\mathrm{c}
$$

Taxonomy of Fractal Geometry .The fractal geometry is classified by connectivity properties and fractal constructive methods (e.g., IFS, Lindenmayer-system, etc.) The fractal geometry includes dust (totally disconnected, cantor sets,) dendrites (singly connected throughout, without loops,) multiply connected (connected with loops,) and hybrids (infinitely many components each containing a curve.) In this part, the basis of the condition for manufacturability, initiator of fractals (the initiator itself is essentially "iteration") are defined. Actually, fractal objects (e.g. natural objects) modeled for physical making have to be in finite form. Therefore, the intermediate state of fractal generated model will be the main focus, such model is also called prefractal. Six different domains can deal with prefractals, they include visibility along a line, visibility from a point, visibility from a straight line segment, visibility from a polygon, visibility from a region and global visibility. The problem domains can further be categorized as discrete or continuous. The geometries of the prefractals are discussed in Fig. 1 according to their initiators.

\section{Manufacturability of Fractal Geometry}

Manufacturability of Fractal Geometry Using Traditional Manufacturing Process. At present, manufacturing techniques can be classified into three categories. They are forming processes, material removal processes and material addition processes. Forming processes such as injection moulding, die casting, and stamping account for a very high percentage of parts that are manufactured worldwide. Material removal processes such as computer numerical control (CNC) machining and electrical discharge machining (EDM) start from a larger amount of bulk material and remove all excess material. They are commonly used in mould making. All these processes are well suited for mass production. However, these processes can hardly be used to make three-dimensional (3D) physical aesthetic appealing objects with complex shape. In this part, we will discuss why these common techniques cannot be used to manufacture the 3D physical fractal objects. 


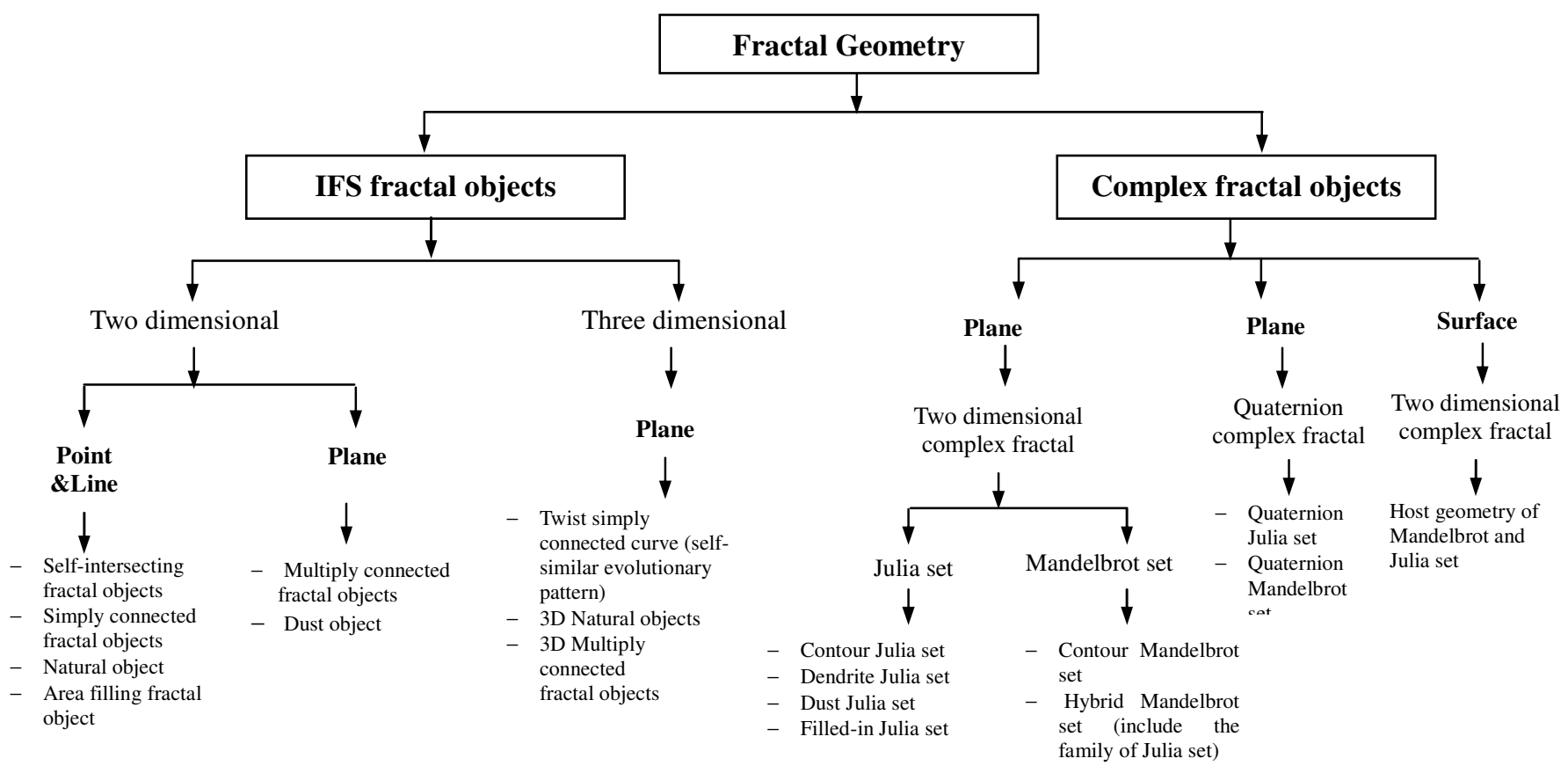

Fig.1 Taxonomy of fractal objects by initiator of fractals

The conventional manufacturing processes mainly include point visible (Fig. 2,) line visible (Fig. 3) and surface visible process (Fig. 4.) NC machining is said to be process that requires point visibility which is short for point-point visibility. For example, turning machine can produce a model with symmetrical geometry, but they cannot produce fractal object with symmetrical geometry (quaternion complex fractal.) The reason is that the fractal object has with fragmented (disconnected) features. Another example is that drilling machine can remove the internal feature of objects, but it is difficult to remove the intricate shape of multiply IFS fractals (e.g. Sierpinski tetrahedron) with complex features. Only 5-axis machining can manufacture host geometry which is a fractal pattern for engraving or embossing by projecting a fractal curve onto a free-form surface. And, the host geometry of fractals can be generated using existing computer-aided design (CAD) software (e.g. PowerSHAPE, ArtCAM, etc.) However, the fractal geometry cannot communicate with the CAD system efficiently as the CAD system considers only Euclidean geometry. For line visible process (e.g. wire-cutting electric-discharge machining (EDM), ) there must be line visibility between the effector and the workpiece such that all the points in a segment of the cutting wire are visible to the corresponding points in a line segment on the workpiece. Die sinking EDM, sheet-metal stamping, die casting and plastic mould injection demand surface visibility. Both line and surface visible process also cannot manufacture fractals with fragmented features and complex intricate features.

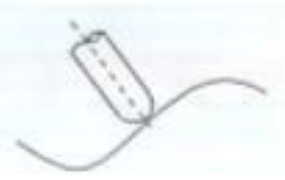

Fig.2 Point visibility

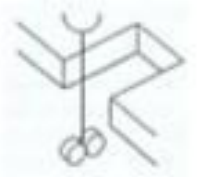

Fig.3 Line visibility

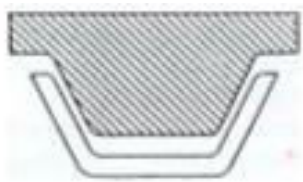

Fig.4 Surface visibility

Manufacturability of Fractal Geometry Using Layer Manufacturing Process. Today, we also have a new group of manufacturing techniques in which the physical object is produced by adding rather than removing or deforming material. It is the layer manufacturing (LM) technology, also known 
as solid freeform fabrication (SFF, ) computer automated manufacturing or rapid prototyping (RP.) LM technology belongs to plane visibility for an entire layer. The LM device can be conceptualized as a planar source, giving rise to point-by-point correspondence between the source and the target. Therefore, it offers advantages in many applications compared to classical subtractive fabrication methods such as milling or turning. Objects can be formed with any geometric complexity or intricacy without the need for elaborating machine setup or final assembly. Therefore, LM is a potential candidate for making the aesthetically appealing objects physically. The CAD technology inputs Euclidean geometry for LM. 3D physical Euclidean objects are then constructed layer by layer using materials such as amorphous plastic and wax. It has been noted that the use of LM in manipulating complex shape objects is very limited. Therefore, we will base on LM materials (e.g., thermoplastic, sand, metal, elastomer, etc.,) process types (e.g., photopolymer solidification, material deposition, powder solidification, lamination, etc.) to analyze the manufacturability of fractal geometry using LM techniques, as well as an attempt to explore the limits of the technologies.

\section{Limitation of LM Technology}

In fact, fractals can be manufactured by many types of LM machines. However, fractal objects with fragmented features can only be produced by using photopolymer solidification (e.g. 3D system, stereolithography (SL)) as it has pre-selected a red-tint function [4] (Fig. 5.) Unless, the user can supply a host geometry (Fig. 6) for disconnected fractal models to attach. Besides, only 3D color printers can produce multi-color objects (Fig.7.) These machines are capable of producing model in full color by installing colored inkjet print heads.

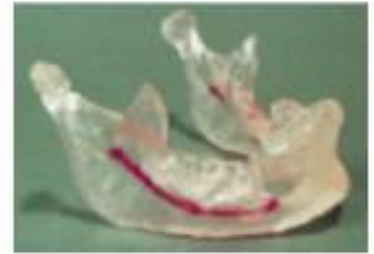

Fig.5 A colored physical model

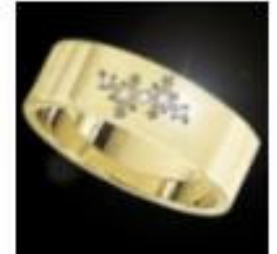

Fig.6 Fractal on host geometry

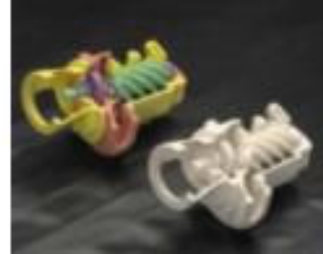

Fig.7 Monochrome and colored prototype

Moreover, material deposition (e.g. fused deposition modelling (FDM)) (Fig. 8) is not suitable to manufacture self-intersecting fractal objects due to the problem of material overlap (Fig. 9, Fig. 10.)

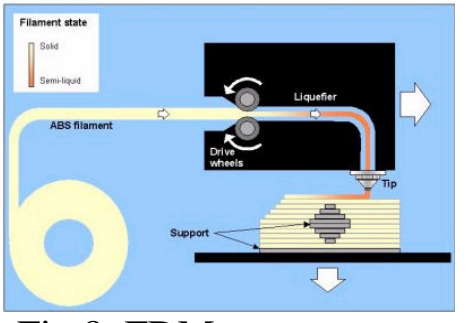

Fig.8 FDM process

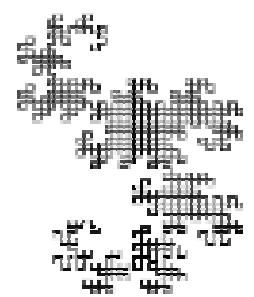

Fig.9 Dragon curve

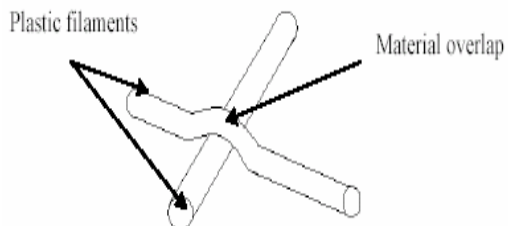

Fig.10 Material overlap of self-intersecting curve

Another problem is the manufacturing multiply connected IFS fractals (Fig.9) Support structures are needed during fabricating for the numerous overhanging geometries or internal features which have to be removed later by breaking them away from the fractals. Photopolymer solidification and material deposition (e.g. FDM) processes' supports are very difficult to remove in the post-processing step. Unless, the FDM use the water-soluble support material to support the free-formed layers and which 
can be dissolved away. On the other hands, lamination processes (e.g. laminated object modeling $(\mathrm{LOM})$ ) without supports required are very difficult to remove the unnecessary material to expose the intrinsic fractal features.

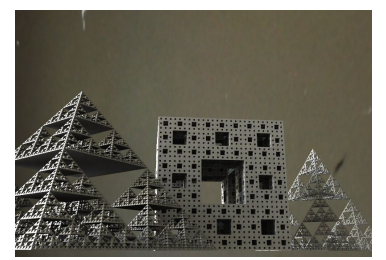

Fig.9 Multiply connected IFS fractals

\section{Conclusions}

In conclusion, fractal can only be manufactured by LM technologies. It is shown that the connectivity of fractal object is important since a simply connected object is more preferred in its physical making. Future works can be extended for layer manufacturing of fractal objects.

\section{Acknowledgements}

The work described in this paper was substantially supported by a grant from the Research Grants Council of the Hong Kong Special Administrative Region (Project No.: B-Q601.)

\section{References}

[1] Y.C. Woo: Computer Aided Design Vol. 26 (1994), p. 6

[2] B. Mandelbrot: The fractal geometry of nature (W.H. Freeman, 1983)

[3] M.F Barnsley and S.Demko: Proc. Roy. Soc. London (1985), p. 243

[4] V. Pattijn, I. Samson, S.J. Vander, et al: Personalized titanium membrane reconstruction in tumor surgery (2000). 